\title{
Investigation of Material Instability Behaviors Caused by Combined Stress Loadings
}

\author{
Wei Ma \\ Institute of Mechanics, Chinese Academy of Sciences, Beijing, China
}

Keywords: material instability criterion, thermoviscoplasticity, instability phase diagram, combined stress state, numerical simulation.

\begin{abstract}
This study involves the stability of plastic flow of thermoviscoplastic materials. The general instability criterion is proposed for determining the onset conditions of instability and the transition conditions among various plastic deformation behaviors. By using the phase diagram method, the transition of material instability modes under complex loading conditions is described and the analytical results are further validated with numerical examples.
\end{abstract}

\section{Introduction}

Instability often occurs prior to ultimate failure of materials, and thus the prediction of the onset and evolvement of instability is necessary for evaluating the mechanical behaviors of materials. Necking is well known as instability behavior of materials. Many studies on necking show that, when the stretch-rate governs plastic flow behaviors, the strain-rate, inertia and material properties may cause the necking retardation $[1,2]$. For the bodies with different geometrical configurations, the strain-rate substantially increases the strain prior to necking [3]. Moreover, necking onset and evolution strongly depends on the material constitutive behaviors, the innate characteristic lengths and imperfection inside materials [4-6]. Shear band is another instability behavior of materials. It is well known that the shear band instability may be caused by the bifurcation from homogeneous deformation to shear localization [7]. The strain rate sensitivity, strain hardening and thermal softening, inertia and heat conduction [8-10] affect the formation of shear bands. Moreover, the shear localization is quite sensitivity to material inhomogeneity and imperfection $[11,12]$.

To evaluate instability behaviors as necking or shear banding, various criteria have been proposed in the previous studies. However, a universal criterion that may evaluate different instability behaviors caused by combined stress loadings is still lacking.
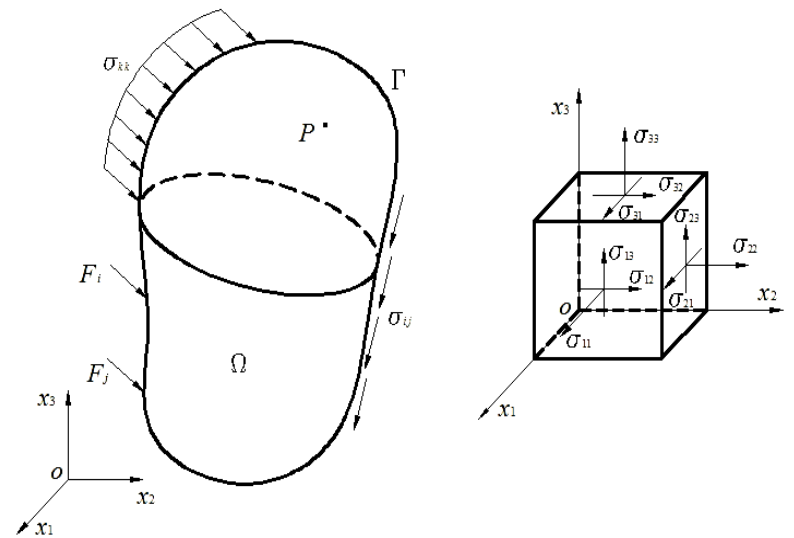

Fig.1 The configuration with general loading conditions and the stress state used in this study.

This work proposes a universal criterion and studies the influences of material mechanical behaviors and loading conditions on the stability of plastic deformation with phase diagram. Afterwards, for validating the analytic results, a numerical example is provided and conclusions are summarized.

\section{Theory}

Consider a thermoviscoplastic body that occupies a spatial region $\Omega$ with external surface $\Gamma$ and is subjected to general loadings (Fig. 1). A rectangular coordinate system labeled $x_{1}, x_{2}, x_{3}$ is introduced so that the vector $\mathbf{x}=\mathbf{x}\left(x_{1}, x_{2}, x_{3}\right)$ denotes the position of a particle in the body in the current 
configuration at time $t$. The stress state of any point in the body is also shown in this figure. Let $\sigma_{i j}$ denote the Cauchy stress tensor, $\varepsilon_{i j}$ the strain tensor, $\dot{\varepsilon}_{i j}$ the strain rate tensor, and $T(\mathbf{x}, t)$ the absolute temperature. The equation of motion without body forces is

$$
\frac{\partial \sigma_{i j}}{\partial x_{j}}=\rho \frac{\partial^{2} u_{i}}{\partial t^{2}}
$$

and the energy balance equation is

$$
\rho c \frac{\partial T}{\partial t}=\lambda \nabla^{2} T+\Phi \sigma_{i j} \dot{\varepsilon}_{i j}
$$

where $\rho$ is the mass density, $c$ the specific heat, $\lambda$ the thermal conductivity, $\nabla^{2}$ the Laplacian operator and $\Phi$ the fraction of plastic work that is converted to heat with value of 0.93 . Moreover, to ensure the continuity of plastic deformation, the compatibility conditions required are

$$
e_{i k m} e_{j l n} \varepsilon_{i j, k l}=0
$$

Assume that the material is incompressible and isotropic, and then the general form of thermoviscoplastic constitutive equation is

$$
\sigma_{i j}=\sigma\left(\varepsilon_{i j}, \dot{\varepsilon}_{i j}, T\right)
$$

Through the linear perturbation analysis of govern equations (1)-(3), we get the following characteristic equation

$$
\begin{aligned}
& -C_{13} C_{22} \alpha^{3}+\left[\left(C_{12} C_{24}-C_{13} C_{23}\right) D_{1} k^{2}-C_{13} C_{22} C_{31} k^{2}-C_{13} C_{22}\left(D_{2}+D_{3}\right)\right] \alpha^{2} \\
& +\left[\begin{array}{l}
C_{31}\left(C_{12} C_{24}-C_{13} C_{23}\right) D_{1} k^{2}+\left(C_{12} C_{24}-C_{13} C_{23}\right)+C_{21}\left(C_{12} D_{4}-C_{13} D_{5}\right) \\
+C_{12}\left(C_{21} D_{6}+C_{24} D_{7}+C_{24} D_{8}\right)-C_{13}\left(C_{21} D_{9}+C_{23} D_{7}+C_{23} D_{8}\right)
\end{array}\right] k^{2} \alpha \\
& +C_{31}\left(C_{12} C_{24}-C_{13} C_{23}\right) k^{4}+\left[C_{12}\left(C_{21} D_{10}+C_{24} D_{2}+C_{24} D_{3}\right)-C_{13}\left(C_{21} D_{11}+C_{23} D_{2}+C_{23} D_{3}\right)\right] k^{2}=0
\end{aligned}
$$

The equation (5) is a cubic polynomial of growth rate $\alpha_{0}$, in which the dimensionless variables $\alpha, k$ and constants $C_{i}$ and $D_{i}$ are given in [14]. If (5) has real positive root, we may obtain two functions as

$$
\begin{aligned}
& F_{1}(\alpha)=240\left(\frac{c R_{0}}{\lambda} \alpha+1\right)\left[\alpha+\left(\dot{\gamma}_{120}+\dot{\gamma}_{230}+\dot{\gamma}_{310}+\dot{\varepsilon}_{110}+\dot{\varepsilon}_{220}+\dot{\varepsilon}_{330}\right) \frac{\lambda \Phi P_{0}}{\rho c^{2} Q_{0}}\right] \\
& F_{2}(\alpha)=4\left\{\left(3+5 \frac{c R_{0}}{\lambda}\right) \alpha+5-9\left(\begin{array}{l}
\eta_{11} \sigma_{110}+\eta_{22} \sigma_{220}+\eta_{33} \sigma_{330} \\
+2\left(\eta_{12} \tau_{120}+\eta_{23} \tau_{230}+\eta_{31} \tau_{310}\right)
\end{array}\right) \frac{\Phi P_{0}}{\rho c Q_{0}}-9 D_{E}\left(R_{0}+\frac{\lambda t_{c}}{c}\right) \frac{\Phi P_{0}}{\rho c Q_{0}}\right\}^{2}
\end{aligned}
$$

where the characteristic time $t_{c}=1 / \alpha$ is introduced. $Q_{0}, R_{0}$ and $P_{0}$ denote the strain hardening, strain rate hardening and thermal softening, respectively. If $F_{2}(0)>F_{1}(0)$, the positive value of $\alpha$ exists constantly. Thus, we obtain the onset condition of instability as

$$
\begin{aligned}
& \frac{9 \Phi P_{0}}{5}\left[2\left(\eta_{12} \tau_{120}+\eta_{23} \tau_{230}+\eta_{31} \tau_{310}\right)+\left(\eta_{11} \sigma_{110}+\eta_{22} \sigma_{220}+\eta_{33} \sigma_{330}\right)+D_{E}\left(R_{0}+\frac{\lambda t_{c}}{c}\right)\right] \\
& -2 \sqrt{\frac{3}{5}\left(\dot{\varepsilon}_{110}+\dot{\varepsilon}_{220}+\dot{\varepsilon}_{330}+\dot{\gamma}_{120}+\dot{\gamma}_{230}+\dot{\gamma}_{310}\right) \rho \lambda \Phi P_{0} Q_{0}}>\rho c Q_{0}
\end{aligned}
$$

where $\lambda$ is thermal conductivity, $D_{E}=D_{E S}+D_{E T}$ and

$$
\begin{aligned}
& D_{E S}=\left(2 \eta_{12}-5 / 9\right) \dot{\gamma}_{120}+\left(2 \eta_{23}-5 / 9\right) \dot{\gamma}_{230}+\left(2 \eta_{31}-5 / 9\right) \dot{\gamma}_{310} \\
& D_{E N}=\left(\eta_{11}-5 / 9\right) \dot{\varepsilon}_{110}+\left(\eta_{22}-5 / 9\right) \dot{\varepsilon}_{220}+\left(\eta_{33}-5 / 9\right) \dot{\varepsilon}_{330}
\end{aligned}
$$

The function $D_{E}$ has the dimension of strain rates. In the plane loading case, the instability criterion is

$$
2 \Phi P_{0}\left[2 \tau_{i j 0}+\sigma_{i i 0}+\sigma_{j j 0}-\left(\dot{\varepsilon}_{i i 0}+\dot{\varepsilon}_{i j 0}-\dot{\gamma}_{i j 0}\right)\left(R_{0}+\frac{\lambda t_{c}}{c}\right)\right]-2 \sqrt{6 \rho \lambda \Phi P_{0} Q_{0}\left(\dot{\gamma}_{i j 0}+\dot{\varepsilon}_{i i 0}+\dot{\varepsilon}_{j j 0}\right)}>3 \rho c Q_{0}
$$

In simple shear case, the shear localization instability criteria are

$$
\Phi P_{0} \tau_{i j 0}-2 \sqrt{\rho \lambda \Phi P_{0} Q_{0} \dot{\gamma}_{i j 0}}>\rho c Q_{0}
$$




\section{Results and Discussion}

The conditions (7) and (9) indicate whether shear localization instability of materials takes place or nonlocalization thermal softening instability depends on loading conditions; whereas, the condition (10) characterizes whether shear localization instability takes place or not is governed only by shear stresses. Physically, these criteria show that the material instability takes place once the thermal softening of plastic deformation surpasses the work hardening. From the mathematical expressions, we can explicitly see the influences of various physical quantities on thermal softening of materials. The stresses constantly enhance the thermal softening effect and make the plastic flow tend to instable. The effects of strain rates, strain rate sensitivity $R_{0}$ and heat conductivity $\lambda$ on thermal softening depend on the proportion factors $\eta_{i j}$. Thus, the criteria may estimate the instability behaviors for rate-dependent materials $\left(R_{0} \neq 0\right)$, rate-independent materials $\left(R_{0}=0\right)$, conductive materials $(\lambda \neq 0)$ and non-conductive materials $(\lambda=0)$, respectively. The term involving the product of thermal conductivity $\lambda$ and characteristic time $t_{c}$ characterizes the influence of heat conduction on the instability of materials. Therefore, both of the critical conditions (7) and (9) are universal criteria, which not only consider the effects of strain hardening, strain rate sensitivity and thermal softening on the instability behaviors, but also take into the effects of normal stresses, strain rates and heat conduction account. The modes of material instability may be the adiabatic shear localization or the non-localized thermal softening. From the criteria, we can get the relevant criteria to estimate the instability behaviors of materials for the quasi-static or dynamic loadings, uniaxial tension or compression loadings, homogeneous or inhomogeneous simple shear loadings, tension- or compression-shear combined stress loadings and so on [13].

Fig. 2 is the instability phase diagram based on the functions $F_{1}(\alpha)$ and $F_{2}(\alpha)$. Fig. 2a corresponds to the case of the tension-shear combined stress loading. The interval $\left(P_{1}, P_{2}\right)$ confirms the critical conditions of localization shear instability and the intervals outside $\left(P_{1}, P_{2}\right)$ determine nonlocalization thermal softening instability conditions. Thus, the phase points $P_{1}$ and $P_{2}$ denote the upper limits of the shear localization instability and nonlocalization instability conditions, respectively and the phase points in $\left(P_{1}, P_{2}\right)$ as $P_{3}$ denote that material plastic flow may go through shear localization instability.

When $30 \%$ of total plastic work is dissipated by tensile deformation, the phase point $P_{4}$ falls on the point $P_{2}$. Hence, the point $P_{4}$ denotes a critical condition of instability mode transition. If the dissipative rates of tension deformation increase, the phase point $P_{i}$ gets into the interval $\left(P_{4}, P_{5}\right)$, which denotes the nonlocalization thermal softening instability conditions because that, in this interval, tension deformation is the dominated mechanism of plastic flow and the thermal softening caused by shear deformation just assists the nonlocalization instability behaviors. At the left end of the interval $P_{5}$, the tensile deformation dissipates $60 \%$ of total plastic work. We have seen that, in tension-shear combined stress loading case, instability certainly occur. Fig. $2 b$ shows the phase diagrams of compression-shear combined stress loading case. The entire phase plane is divided into three parts. The phase points, as $P_{2}$, in the interval $\left(P_{3}, P_{1}\right)$ denote the localization shear instability conditions. The right end $P_{1}$ denotes the upper limit of the localization shear instability conditions and the left end $P_{3}$ with plastic work rate ratio 14:11 confirms the critical condition that estimates stable and unstable plastic flow behaviors of materials. When the plastic work rate dissipated by compressive deformation is larger than $56 \%$ of total plastic work, materials get into steady plastic flow state $(\alpha<0)$ if the compressive deformation induces the strain hardening of materials $\left(Q_{0}>0\right)$; otherwise, nonlocalization thermal softening instability as denoted by $P_{4}$ is possible if the compressive deformation induces strain softening of materials $\left(Q_{0}<0\right)$. Therefore, the interval $\left(P_{1}, \infty\right)$ on the right-hand side $P_{1}$ defines the nonlocalization thermal softening instability conditions.

Fig. 3 and Fig. 4 show the simulating results of material plastic deformation and temperature distributions under the tension-shear combined stress loading conditions. When the applied tangential velocity is $4 \mathrm{~m} / \mathrm{s}$ and the normal velocity is $1 \mathrm{~m} / \mathrm{s}$, the contour in Fig. 3a shows that the plastic shear strain is confined in a narrow zonal region of testing segment, implying that the localized plastic deformation has fully developed. The contour of temperature in Fig. $3 \mathrm{~b}$ shows that the temperature in 
the region of localization deformation of materials increases sharply. The peak temperature is close to $1500{ }^{\circ} \mathrm{C}$, suggesting that adiabatic shear bands have developed. When the applied tangential velocity is $3 \mathrm{~m} / \mathrm{s}$ and the normal velocity is $2 \mathrm{~m} / \mathrm{s}$, the contour of plastic shear strain in Fig. 4a shows that the plastic flow of materials spread out in the entire testing segment of simulating specimen. Note that the peak strain is merely about 1.2 and the mean strain is about 0.8 , so that no deformation localization develops presently. The contour of temperature in Fig. $4 \mathrm{~b}$ shows that no sharp temperature rise takes place. The heat affecting zone fills up the full testing segment and extends outside the testing segment by heat conduction. The peak temperature is below $300{ }^{\circ} \mathrm{C}$, meaning that the plastic flow does not cause the evident thermal softening in materials currently. Consequently, the plastic deformation of materials may be nonlocalization thermal softening instability or long range plastic flow. The results coincide with the theoretical prediction of Fig. 2 a.

Similarly, for the situations of compressive-shear combined stress loadings, when the dissipative rate of plastic work in compressive deformation is equal to the $40 \%$ of total dissipative rate, the contours of plastic shear strain and temperature shown in Fig. 5 demonstrate that the shear localization deformation in materials has developed. When the dissipative rate of plastic work in compressive deformation is equal to the $60 \%$ of total dissipative rate, the simulating results as shown in Fig. 6 denote that the nonlocalization thermal softening instability is possible. The results agree with the theoretical prediction of Fig. $2 b$.
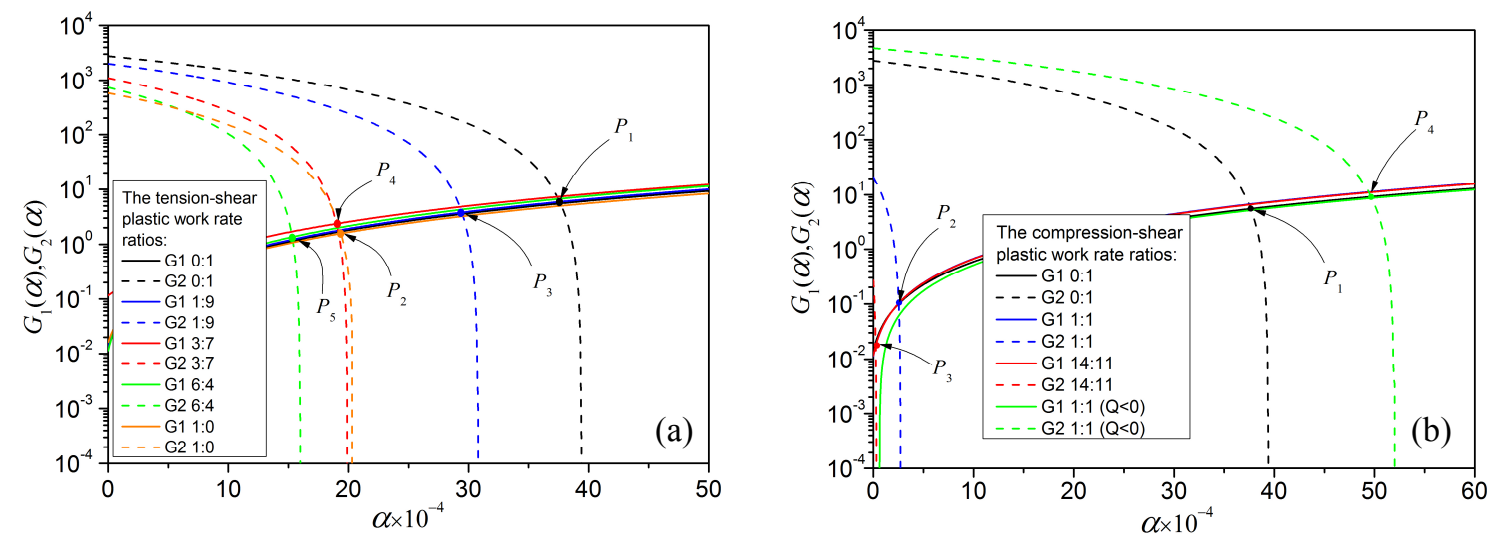

Fig.2. The phase diagrams of the tension-shear (a) and compression-shear (b) in combined stress loading case.

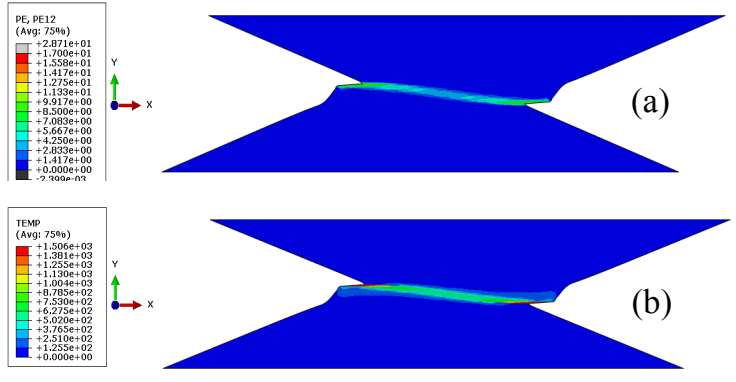

Fig. 3. The contours of plastic shear strain (a) and temperature (b) when the applied tangential velocity is $4 \mathrm{~m} / \mathrm{s}$ and the normal velocity is $1 \mathrm{~m} / \mathrm{s}$.

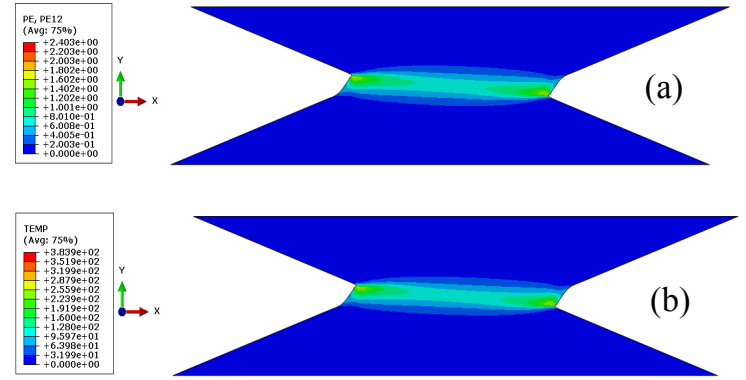

Fig. 4. The contours of plastic shear strain (a) and temperature (b) When the applied tangential velocity is $3 \mathrm{~m} / \mathrm{s}$ and the normal velocity is $2 \mathrm{~m} / \mathrm{s}$. 


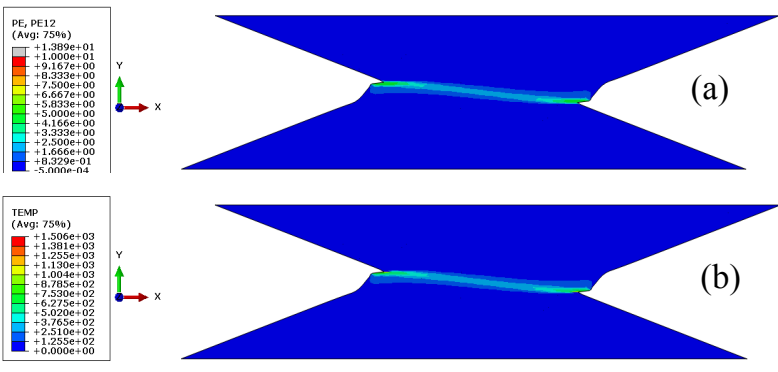

Fig. 5. The contours of plastic shear strain (a) and temperature (b) when the tangential velocity is $3 \mathrm{~m} / \mathrm{s}$ and the normal velocity is 2 $\mathrm{m} / \mathrm{s}$.

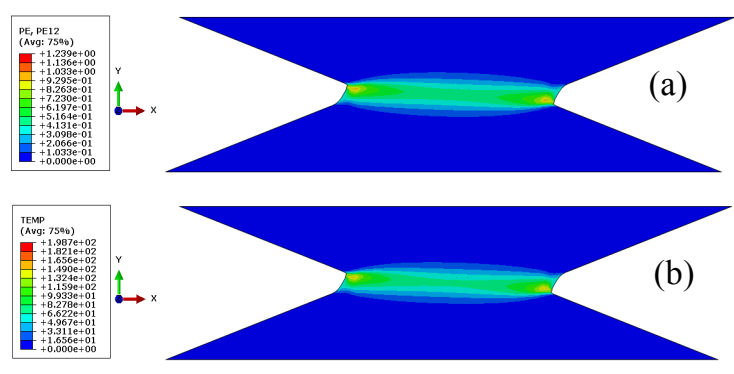

Fig. 6. The contours of plastic shear strain (a) and temperature (b) when the tangential velocity is $2 \mathrm{~m} / \mathrm{s}$ and the normal velocity is 3 $\mathrm{m} / \mathrm{s}$.

\section{Summary}

A universal criterion is obtained to estimate materials instability behaviors. The criterion indicates the effects of strain hardening, strain rate sensitivity, thermal softening and heat conduction as well as normal and shear stresses and strain rates on instability behaviors of materials. Physically, it shows that plastic flow loses its stability once the thermal softening surpasses the work hardening. The results also reveal that the effects of strain rate sensitivity and heart conduction on thermal softening are related with various strain rates. The numerical results further show that the criterion may be used to judge the stable or instable behaviors of plastic flow.

\section{Acknowledgement}

The authors acknowledge financial supports from the National Nature Science Foundation of China (Grant No. 10972227 and 11132011) and the National Key Basic Research Program of China (Grant No. 2009CB724401).

\section{References}

[1] J. W. Hutchinson, H. Obrecht, Tensile instabilities in strain rate dependent materials. In: ICF4, Waterloo, Canada, 1 (1977) 101.

[2] Z. Xue, A. Vaziri, J. W. Hutchinson, Material aspects of dynamic neck retardation. J. Mech. Phys. Solids. 56 (2008) 93.

[3] J. W. Hutchinson, K. W. Neale, Influence of strain rate sensitivity on necking under uniaxial tension. Acta Metal., 25 (1977) 839.

[4] V. B. Shenoy, L.B. Freund, Necking bifurcations during high strain rate extension J. Mech. Phys. Solids, 47 (1999) 2209.

[5] P. Hähner, A generalized criterion of plastic instability and its application to creep damage and superplastic flow. Acta metall, mater. 43 (1995) 4093.

[6] R. Hill On the mechanics of localized necking in anisotropic sheet metals. J. Mech. Phys. Solids, 49 (2001) 2055.

[7] Rudnicki and Rice J. W. Rudnicki, J. R. Rice, Conditions for the localization of deformation in pressure sensitive dilatants materials. J. Mech. Phys. Solids, 23 (1975) 371.

[8] R. J. Clifton, Material response to ultra-high loading rates. NRC Report NMAB-356, 1980, 129.

[9] Y. L. Bai, Thermo-plastic instability in simple shear. J. Mech. Phys. Solids, 30 (1982)195.

[10] J. Pan, Perturbation analysis of shear strain localization in rate sensitive materials. Int. J. Solids Struct. 19 (1983) 153.

[11] J. W. Hutchinson, V. Tvergaard, Shear band formation in plane strain. Inter. J. Solids Struct., 17 (1981) 451.

[12] A. Needleman, Dynamic shear band development in plane strain. J. Appl. Mech. 56 (1989) 1.

[13] W. Ma, X. W. Li, L. H. Dai, Z. Ling, Instability criterion of materials in combined stress states and its application to orthogonal cutting process. Int. J. Plast., 30-31 (2012) 18-40. 
Advances in Engineering Plasticity XI

10.4028/www.scientific.net/KEM.535-536

\section{Investigation of Material Instability Behaviors Caused by Combined Stress Loadings}

10.4028/www.scientific.net/KEM.535-536.168

\section{DOI References}

[2] Z. Xue, A. Vaziri, J. W. Hutchinson, Material aspects of dynamic neck retardation. J. Mech. Phys. Solids. 56 (2008) 93.

http://dx.doi.org/10.1016/j.jmps.2007.04.003 